\title{
Distributed Energy-Efficient Cooperative Routing in Wireless Networks
}

\author{
Ahmed S. Ibrahim, Zhu Han†, and K. J. Ray Liu \\ Department of Electrical and Computer Engineering, University of Maryland, College Park, MD 20742, USA \\ $\nmid$ †epartment of Electrical and Computer Engineering, Boise State University, Boise, ID 83725, USA
}

\begin{abstract}
Recently, cooperative routing in wireless networks has gained much interest due to its ability to exploit the broadcast nature of the wireless medium in designing powerefficient routing algorithms. Most of the existing cooperationbased routing algorithms are implemented by finding a shortestpath route first. As such, these routing algorithms do not fully exploit the merits of cooperative communications at the physical layer. In this paper, we propose a cooperation-based routing algorithm, namely, Minimum Power Cooperative Routing (MPCR) algorithm, which makes full use of the cooperative communications while constructing the minimum-power route. The MPCR algorithm constructs the minimum-power route as a cascade of the minimum-power single-relay building blocks from the source to the destination. Hence, any distributed shortestpath algorithm can be utilized to find the optimal route with polynomial complexity, while guaranteeing certain throughput. We show that the MPCR algorithm can achieve power saving of $57.36 \%$ compared to the conventional shortest-path routing algorithms. Furthermore, the MPCR algorithm can achieve power saving of $37.64 \%$ compared to the existing cooperative routing algorithms, in which the selected routes are constructed based on the noncooperative routes.
\end{abstract}

\section{INTRODUCTION}

In wireless networks such as ad hoc networks, nodes spend most of their power in communication, either sending their own data or relaying other nodes' data [1]. Therefore, designing power-efficient routing algorithms is one of the major concerns in wireless networks. Furthermore, the communication power can be reduced by jointly considering other layers' protocols, which make use of the broadcast nature of the wireless medium. Moreover, these algorithms should be implemented in a distributed way. Therefore, the main goal of this paper is to design a distributed minimum-power routing algorithm for wireless networks, which exploits the broadcast nature of the wireless medium.

Recently, cooperative communication for wireless networks has gained much interest due to its ability to mitigate fading through achieving spatial diversity, while resolving the difficulties of installing multiple antennas on small communication terminals. In cooperative communications, relays are assigned to help a sender in forwarding its information to its receiver. Thus, the receiver gets several replicas of the same information via independent channels. Various cooperative diversity protocols were proposed and analyzed in [2]-[10].

The classical relay channel model based on additive white Gaussian noise (AWGN) channels was presented in [2]. In [3], Laneman et al. described various techniques of cooperative communication, such as decode-and-forward, amplify-andforward, selection relaying, and incremental relaying. In [4], a distributed space-time coded (STC) cooperative scheme was proposed by Laneman et al. In [5] and [6], Sendonaris et al. introduced user cooperation diversity. A two-user CDMA cooperative system, where both users are active and use orthogonal codes, was implemented in this two-part series. In [7],[8], relay-selection schemes for single- and multi-node decode-and-forward cooperative systems were proposed. In [9], the authors have provided SER performance analysis for the decode-and-forward multi-node scheme. Finally, a distributed relay-assignment algorithm for wireless communications has been proposed in [10].

The merits of the cooperative communications in the physical layer have been explored. However, the impact of the cooperative communications on the design of the higher layers is not well-understood yet. Routing algorithms, which are based on the cooperative communications and known as cooperative routing [11], is an interesting research area and can lead to significant power savings. The cooperative routing proposed in [11] makes use of two facts: the Wireless Broadcast Advantage (WBA) in the broadcast mode and the Wireless Cooperative Advantage (WCA) in the cooperative mode. In the broadcast mode each node sends its data to more than one node, while in the cooperative mode many nodes send the same data to the same destination.

The cooperative routing problem has been recently considered in the literature [11]-[15]. In [11], the optimum route is found through a dynamic programming algorithm. In [12], the minimum-power route is chosen while guaranteeing fixed transmission rate. In [13], Li et al. proposed the Cooperative Shortest Path (CSP) algorithm, which chooses the next node in the route that minimizes the power transmitted by the last $L$ nodes added to the route. Sikora et al. presented in [14] an information-theoretic viewpoint of the cooperative routing in linear wireless network for both the power-limited and bandwidth-limited regimes. In addition, the authors in [14] analyzed the transmitted power, required to achieve a desired end-to-end rate. In [15], the authors proposed three cooperative routing algorithms, namely, relay-by-flooding, relay-assisted routing, and relay-enhanced routing.

Most of the existing cooperation-based routing algorithms are implemented by finding a shortest-path route first. Since the cooperative route is based on the shortest-path one, these routing algorithms do not fully exploit the merits of cooperative communications at the physical layer. This is our main motivation to propose a cooperation-based routing algorithm that takes into consideration the effect of the cooperative communications while constructing the minimum-power route.

In this paper, we consider the minimum-power routing problem with cooperation in wireless networks. The optimum route is defined as the route that requires the minimum transmitted power while guaranteeing certain Quality of Service (QoS). 
The QoS is characterized by the end-to-end throughput. We derive a cooperation-based link cost formula, which represents the minimum transmitted power that is required to guarantee the desired QoS over a particular link. The main contribution of this paper is the proposed cooperation-based routing algorithm, namely the Minimum Power Cooperative Routing (MPCR) algorithm, which can choose the minimum-power route while guaranteeing the desired QoS. It will be shown that the MPCR algorithm can achieve power saving of $57.36 \%$ compared to the conventional shortest-path routing algorithms. Furthermore it can achieve power saving of $37.64 \%$ with respect to the Cooperation Along the Shortest Non-Cooperative Path (CASNCP) algorithm, which finds the shortest-path route first then it applies the cooperative communication upon the constructed route to reduce the transmitted power.

The rest of the paper is organized as follows. In the next section, we formulate the minimum-power routing problem. In addition, we describe the network model and derive closedform expressions for the minimum transmitted power per hop in Section II. We describe two cooperation-based routing algorithms in Section III. In Section IV, we show the numerical results for the power savings of the proposed algorithm. Finally, Section V concludes the paper.

\section{System Model AND Link Analysis}

In this section, we describe the network model and formulate the minimum-power routing problem. Then, we present the direct transmission and cooperative transmission modes. Finally, we derive the required power for these two transmission modes in order to achieve certain throughput.

\section{A. Network Model}

We consider a graph $G(N, E)$ with $N$ nodes and $E$ edges. Given any source-destination pair $(S, D) \in\{1, \ldots, N\}$, the goal is to find the $S-D$ route that minimizes the total transmitted power, while satisfying a specific throughput. For a given source-destination pair, denote $\Omega$ as the set of all possible routes, where each route is defined as a set consisting of its hops. For a route $\omega \in \Omega$, denote $\omega_{i}$ as the $i$-th hop of this route. Thus, the problem can be formulated as

$$
\min _{\omega \in \Omega} \sum_{\omega_{i} \in \omega} P_{\omega_{i}} \text { s.t. } \quad \eta_{\omega} \geq \eta_{o},
$$

where $P_{\omega_{i}}$ denotes the transmitted power over the $i$-th hop, $\eta_{\omega}$ is the end-to-end throughput, and $\eta_{o}$ represents the minimum desired value of the end-to-end throughput. Let $\eta_{\omega_{i}}$ denote the throughput of the $i$-th hop, which is defined as the number of successfully transmitted bits per second per hertz $(\mathrm{b} / \mathrm{s} / \mathrm{Hz})$ of a given hop. Furthermore, the end-to-end throughput of a certain route $\omega$ is defined as the minimum of the throughput values of the hops constituting this route, i.e.,

$$
\eta_{\omega}=\min _{\omega_{i} \in \omega} \eta_{\omega_{i}} .
$$

It has been proven in [13] that the Minimum Energy Cooperative Path (MECP) routing problem, i.e., to find the minimum-energy route using cooperative radio transmission, is

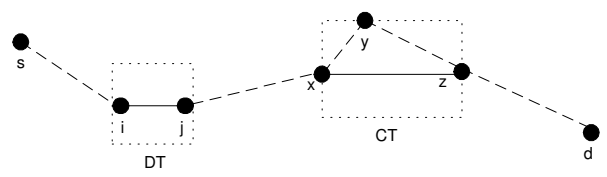

Fig. 1. Cooperative Transmission (CT) and Direct Transmission (DT) modes as building blocks for any route.

NP-complete. This is due to the fact that the optimal path could be a combination of cooperative transmissions and broadcast transmissions. Therefore, we consider two types of building blocks: direct transmission (DT) and cooperative transmission (CT) building blocks. In Fig. 1 the DT block is represented by the link $(i, j)$, where node $i$ is the sender and node $j$ is the receiver. In addition, the CT block is represented by the links $(x, y),(x, z)$, and $(y, z)$, where node $x$ is the sender, node $y$ is a relay, and node $z$ is the receiver. The route can be considered as a cascade of any number of these two building blocks, and the total power of the route is the summation of the transmitted powers along the route. Thus, the minimization problem in (1) can be solved by applying any distributed shortest-path routing algorithm such as the Bellman-Ford algorithm [16].

\section{B. Direct and Cooperative Transmission Modes}

Let $h_{u, v}, d_{u, v}$, and $n_{u, v}$ represent the channel coefficient, length, and additive noise of the link $(u, v)$, respectively. For the direct transmission between node $i$ and node $j$, the received symbol can be modeled as

$$
r_{i, j}^{D}=\sqrt{P^{D} d_{i, j}^{-\alpha}} h_{i, j} s+n_{i, j}
$$

where $P^{D}$ is the transmitted power in the direct transmission mode, $\alpha$ is the path loss exponent, and $s$ is the transmitted symbol.

For the cooperative transmission, we consider a modified version of the decode-and-forward incremental relaying cooperative scheme, proposed in [3]. The transmission scheme for a sender $x$, a relay $y$, and a receiver $z$, can be described as follows. The sender sends its symbol in the current time slot. Due to the broadcast nature of the wireless medium, both the receiver and the relay receive noisy versions of the transmitted symbol. The received symbols at the receiver and the relay can be modeled as

$$
r_{x, z}^{C}=\sqrt{P^{C} d_{x, z}^{-\alpha}} h_{x, z} s+n_{x, z},
$$

and

$$
r_{x, y}^{C}=\sqrt{P^{C} d_{x, y}^{-\alpha}} h_{x, y} s+n_{x, y},
$$

respectively, where $P^{C}$ is the source transmitted power in the cooperative transmission mode.

Once the symbol is received, the receiver and the relay decode it. We assume that the relay and the receiver decide that the received symbol is correctly received if the received signal-to-noise ratio (SNR) is greater than a certain threshold, which depends on the transmitter and the receiver structures. Such system suffers from error propagation but its effect can be neglected. The rationale behind this is that when the relays operate in a high SNR regime, the dominant source of error is 
the channel being in outage, i.e., deep fade, which corresponds to the SNR falling below some threshold. This result has been proven in [17].

If the receiver decodes the symbol correctly, then it sends an acknowledgment (ACK) to the sender and the relay to confirm a correct reception. Otherwise, it sends a negative acknowledgment (NACK) that allows the relay, if it received the symbol correctly, to transmit this symbol to the receiver in the next time slot. This model represents a modified form of the Automatic Repeat Request (ARQ), where the relay retransmits the data instead of the sender, if necessary. The received symbol at the receiver can be written as

$$
r_{y, z}^{C}=\sqrt{P^{C} d_{y, z}^{-\alpha}} h_{y, z} s+n_{y, z} .
$$

In general, the relay can transmit with a power that is different from the sender power $P^{C}$. However, this complicates the problem of finding the minimum-power formula, as will be derived later. For simplicity, we consider that both the sender and the relay send their data employing the same power $P^{C}$.

In this paper, flat quasi-static fading channels are considered, hence, the channel coefficients are assumed to be constant during a complete frame, and may vary from a frame to another. We assume that all the channel terms are independent complex Gaussian random variables with zero mean and unit variance. Finally, the noise terms are modeled as zero-mean, complex Gaussian random variables with equal variance $N_{0}$.

\section{Link Cost Formulation}

Since the throughput is a continuous monotonously-increasing function of the transmission power, the optimization problem in (1) has the minimum when $\eta_{\omega}=\eta_{o}, \forall \omega \in \Omega$. Since the endto-end throughput $\eta_{\omega}=\min _{\omega_{i} \in \omega} \eta_{\omega_{i}}$, then the optimum power allocation, which achieves a desired throughput $\eta_{o}$ along the route $\omega$, forces the throughput at all the hops $\eta_{\omega_{i}}$ to be equal to the desired one, i.e.,

$$
\eta_{\omega_{i}}=\eta_{o}, \quad \forall \omega_{i} \in \omega
$$

This result can be explained as follows. Let $P_{\omega_{1}}^{*}, P_{\omega_{2}}^{*}, \cdots, P_{\omega_{n}}^{*}$ represent the required powers on a route consisting of $n$ hops, where $P_{\omega_{i}}^{*}$ results in $\eta_{\omega_{i}}=\eta_{o}$ for $i=1, \cdots, n$. If we increase the power of the $\mathrm{i}$-th block to $P_{\omega_{i}}>P_{\omega_{i}}^{*}$ then the resulting throughput of the i-th block increases, i.e. $\eta_{\omega_{i}}>\eta_{o}$, while the end-to-end throughput does not change as $\min _{\omega_{i} \in \omega} \eta_{\omega_{i}}=\eta_{o}$. Therefore, no need to increase the throughput of any hop over $\eta_{o}$, which is indicated in (7).

Since the throughput of a given link $\omega_{i}$ is defined as the number of successfully transmitted bits per second per hertz, thus it can be calculated as

$$
\eta_{\omega_{i}}=p_{\omega_{i}}^{S} \times R_{\omega_{i}},
$$

where $p_{\omega_{i}}^{S}$ and $R_{\omega_{i}}$ denote the per-link probability of success and transmission rate, respectively. We assume that the desired throughput can be factorized as

$$
\eta_{o}=p_{o}^{S} \times R_{o},
$$

where $p_{o}^{S}$ and $R_{o}$ denote the desired per-link probability of success and transmission rate, respectively. In the sequel, we calculate the required transmitted power in order to achieve the desired per-link probability of success and transmission rate for both the direct and cooperative transmission modes. We note that the channel gain $\left|h_{u, v}\right|^{2}$ between any two nodes $u$ and $v$, is exponentially distributed with parameter one [18].

For the direct transmission mode in (3), the mutual information between sender $i$ and receiver $j$ can be given by

$$
I_{i, j}=\log \left(1+\frac{P^{D} d_{i, j}^{-\alpha}\left|h_{i, j}\right|^{2}}{N_{0}}\right) .
$$

Without loss of generality, we have assumed unit bandwidth in (10). The outage probability is defined as the probability that the mutual information is less than the required transmission rate $R_{o}$. Thus, the outage probability of the link $(i, j)$ is calculated as

$$
p_{i, j}^{O}=\operatorname{Pr}\left(I_{i, j} \leq R_{o}\right)=1-\exp \left(-\frac{\left(2^{R_{o}}-1\right) N_{0} d_{i, j}^{\alpha}}{P^{o}}\right) .
$$

If an outage occurs, the data is considered lost. The probability of success is calculated as $p_{i, j}^{S}=1-p_{i, j}^{O}$. Thus, to achieve the desired $p_{o}^{S}$ and $R_{o}$ for direct transmission mode, the required transmitted power is

$$
P^{D}=\frac{\left(2^{R_{o}}-1\right) N_{0} d_{i, j}^{\alpha}}{-\log \left(p_{o}^{S}\right)} .
$$

For the cooperative transmission mode, the total outage probability is given by

$$
p_{x, y, z}^{O}=\operatorname{Pr}\left(I_{x, z} \leq R^{C}\right) \cdot \operatorname{Pr}\left(I_{x, y} \leq R^{C}\right)+\operatorname{Pr}\left(I_{x, z} \leq R^{C}\right)
$$$$
\times \quad\left(1-\operatorname{Pr}\left(I_{x, y} \leq R^{C}\right)\right) \times \operatorname{Pr}\left(I_{y, z} \leq R^{C}\right),
$$

where $R^{C}$ denotes the transmission rate for each time slot. In (13), the first term corresponds to the event when both the sender-receiver and the sender-relay channels are in outage, and the second term corresponds to the event when both the sender-receiver and relay-receiver channels are in outage but the sender-relay is not. Consequently, the probability of success of the cooperative transmission mode can be calculated as

$$
\begin{aligned}
p^{S} & =\exp \left(-g d_{x, z}^{\alpha}\right)+\exp \left(-g\left(d_{x, y}^{\alpha}+d_{y, z}^{\alpha}\right)\right) \\
& -\exp \left(-g\left(d_{x, y}^{\alpha}+d_{y, z}^{\alpha}+d_{x, z}^{\alpha}\right)\right),
\end{aligned}
$$

where

$$
g=\frac{\left(2^{R^{C}}-1\right) N_{0}}{P^{C}} .
$$

In (13) and (14), we assume that the receiver decodes the signals received from the relay either at the first time slot or at the second time slot, instead of combining the received signals together. In general, Maximum Ratio Combining (MRC) [19] at the receiver gives a better result. However, it requires the receiver to store an analog version of the received data from the sender, which is not practical. The probability that the source transmits only, denoted by $\operatorname{Pr}(\phi)$, is calculated as

$$
\begin{aligned}
\operatorname{Pr}(\phi) & =1-\operatorname{Pr}\left(I_{x, z} \leq R^{C}\right)+\operatorname{Pr}\left(I_{x, z} \leq R^{C}\right) \operatorname{Pr}\left(I_{x, y} \leq R^{C}\right) \\
& =1-\exp \left(-g d_{x, y}^{\alpha}\right)+\exp \left(-g\left(d_{x, y}^{\alpha}+d_{x, z}^{\alpha}\right)\right),
\end{aligned}
$$


where the term $\left(1-\operatorname{Pr}\left(I_{x, z} \leq R^{C}\right)\right)$ corresponds to the event when the sender-receiver channel is not in outage, while the other term corresponds to the event when both the sender-receiver and the sender-relay channels are in outage. The probability that the relay cooperates with the source is calculated as

$$
\overline{\operatorname{Pr}(\phi)}=1-\operatorname{Pr}(\phi) .
$$

Thus, the average transmission rate of the cooperative transmission mode can be calculated as

$$
R=R^{C} \cdot \operatorname{Pr}(\phi)+\frac{R^{C}}{2} \cdot \overline{\operatorname{Pr}(\phi)}=\frac{R^{C}}{2}(1+\operatorname{Pr}(\phi)),
$$

where $R^{C}$ corresponds to the transmission rate if the sender is sending alone in one time slot and $R^{C} / 2$ corresponds to the transmission rate if the relay cooperates with the sender in the consecutive time slot.

We set the probability of success in (14) as $p^{S}=p_{o}^{S}$ and the average transmission rate in (18) as $R=R_{o}$. By approximating the exponential functions in (14) as $\exp (-x) \approx$ $1-x+x^{2} / 2$, we obtain

$$
g \approx \sqrt{\frac{1-p_{o}^{S}}{d_{e q}}}
$$

where $d_{e q}=d_{x, z}^{\alpha}\left(d_{x, y}^{\alpha}+d_{y, z}^{\alpha}\right)$. Thus, $R^{C}$ can be obtained using (18) as

$$
\begin{aligned}
& R^{C}=\frac{2 R_{o}}{1+\operatorname{Pr}(\phi)} \\
& \approx \frac{2 R_{o}}{2-\exp \left(-\sqrt{\frac{1-p_{o}^{S}}{d_{e q}}} d_{x, y}^{\alpha}\right)+\exp \left(-\sqrt{\frac{1-p_{o}^{S}}{d_{e q}}}\left(d_{x, y}^{\alpha}+d_{x, z}^{\alpha}\right)\right)}
\end{aligned}
$$

where we substituted (19) in (16). In addition, the required power per link can be calculated using (15) and (19) as

$$
P^{C} \approx\left(2^{R^{C}}-1\right) N_{0} \sqrt{\frac{d_{e q}}{1-p_{o}^{S}}} .
$$

Finally, the average transmitted power of the cooperative transmission can be calculated as

$$
P_{\text {avg }}^{C}=P^{C} \cdot \operatorname{Pr}(\phi)+2 P^{C} \cdot \overline{\operatorname{Pr}(\phi)}=P^{C}(2-\operatorname{Pr}(\phi)),
$$

where $\operatorname{Pr}(\phi)$ and $P^{C}$ are given in (16) and (21), respectively.

\section{Cooperation-BAsed Routing Algorithms}

In this section, we propose two cooperation-based routing algorithms, which require polynomial complexity to find the minimum-power route. We assume that each node broadcasts periodically HELLO packet to its neighbors to update the topology information. In addition, we consider a simple Medium Access Control (MAC) protocol, which is the conventional Time Division Multiple Access (TDMA) scheme with equal time slots.

First, we describe the proposed MPCR algorithm for a wireless network of $N$ nodes. The MPCR algorithm can be distributively implemented by the Bellman-Ford shortest path
TABLE I

MPCR Algorithm.

Step 1 Each node $x \in\{1, \ldots, N\}$ behaving as a sender calculates the cost of the its outgoing link $(x, z)$, where $z \in N(x)$ is the receiver as follows. For each other node $y \in N(x), y \neq z$, node $x$ calculates the cost of the cooperative transmission in (22) employing node $y$ as a relay.

Step 2 The cost of the $(x, z)$-th link is the minimum cost among all the costs obtained in Step 1.

Step 3 If the minimum cost corresponds to a certain relay $y *$, node $x$ employs this relay to help the transmission over that hop. Otherwise, it uses the direct transmission over this hop.

algorithm [16]. The derived power formulas for direct transmission and cooperative transmission are utilized to construct the minimum-power route. In the Bellman-Ford shortest path algorithm, each node $i \in\{1, \ldots, N\}$ executes the iteration $D_{i}=\min _{j \in N(i)}\left(d_{i, j}^{\alpha}+D_{j}\right)$, where $N(i)$ denotes the set of neighboring nodes of node $i$ and $D_{j}$ represents the latest estimate of the shortest path from node $j$ to the destination [16], which is included in the HELLO packet. Therefore, the MPCR algorithm is implemented by letting each node calculate the costs of its outgoing links then apply the Bellman-Ford algorithm. Table I describes the MPCR algorithm in details. The worst-case computational complexity of calculating the costs at each node is $O\left(N^{2}\right)$ since it requires two nested loops, and each has the maximum length of $N$ to calculate all the possible cooperative transmission blocks.

Second, we propose a cooperation-based routing algorithm, namely, Cooperation Along the Shortest Non-Cooperative Path (CASNCP) algorithm. The CASNCP algorithm is similar to the heuristic algorithms proposed by Khandani et al. in [11] and Yang et al. in [12] as it applies cooperative communications upon the shortest-path route. However, it is implemented in a different way using the proposed cooperation-based link cost formula. First, it chooses the shortest-path route then it applies the cooperative transmission mode upon each three consecutive nodes in the chosen route; first node as the sender, second node as the relay, and third node as the receiver. Table II describes the CASNCP algorithm.

\section{NuMERICAL RESUltS}

In this section, we present some computer simulations to illustrate the power savings of our proposed MPCR algorithm. We consider a $200 \times 200$ grid, where $N$ nodes are uniformly distributed. The additive white Gaussian noise has variance $N_{0}=-70 \mathrm{dBm}$. Given a certain network topology, we randomly choose a source-destination pair and apply the various routing algorithms, discussed in Section III, to choose the corresponding route. For each algorithm, we calculate the total transmitted power per route. Finally, these quantities are averaged over 1000 different network topologies.

First, we illustrate the effect of varying the desired throughput on the required transmitted power per route. Fig. 2 depicts the transmitted power per route, required by the different routing algorithms for path $\operatorname{loss} \alpha=2$ and $\alpha=4$. As shown, the transmitted power increases with $\alpha$, which is obvious in (12), and can be shown in (22), that the transmitted power is 
TABLE II

CASNCP Algorithm.

Step 1 Implement the Shortest Non-Cooperative Path (SNCP) algorithm using the distributed Bellman-Ford algorithm to choose the conventional shortest-path route $\omega_{S}$ as follows. Each node $i \in\{1, \ldots, N\}$ executes the iteration $D_{i}=$ $\min _{j \in N(i)}\left(d_{i, j}^{\alpha}+D_{j}\right)$, where $N(i)$ denotes the set of neighboring nodes of node $i$ and $D_{j}$ represents the latest estimate of the shortest path from node $j$ to the destination.

Step 2 For each three consecutive nodes on $\omega_{S}$, the first, second, and third nodes behave as the sender, relay, and receiver, respectively, i.e., the first node sends its data to the third node with the help of the second node as discussed in the cooperative transmission mode.

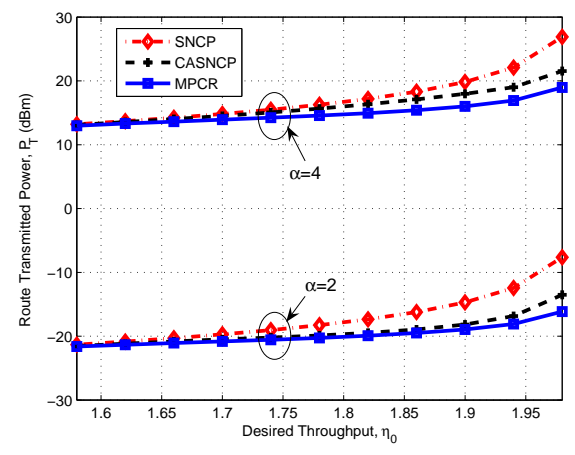

Fig. 2. Required power per route versus the desired throughput for $N=20$ nodes, $N 0=-70 \mathrm{dBm}$, and $R_{d}=2 \mathrm{~b} / \mathrm{s} / \mathrm{Hz}$ in a $200 \times 200$ grid.

proportional to the distance to the power $\alpha$. Since, both cases look similar with a shift in the transmitted power values, we will consider only $\alpha=4$ in the rest of this section as it is more appropriate to represent the wireless medium. It is shown that the SNCP algorithm, which applies the Bellman-Ford shortest-path algorithm, requires the most transmitted power per route. Applying the cooperative communication mode on each three consecutive nodes in the SNCP route results in reduction in the required transmitted power as shown in the CASNCP algorithm's curve. Moreover, the MPCR algorithm requires the least transmitted power among the other routing algorithms.

One of the major results of this paper is that the MPCR algorithm requires less transmitted power than the CASNCP algorithm. Intuitively, this result is because the MPCR applies the cooperation-based link cost formula to construct the minimum-power route. On the contrary, the CASNCP algorithm first constructs shortest-path route then it applies the cooperative communication protocol on the established route. Therefore, the CASNCP algorithm is limited to applying the cooperative-communication protocol on certain number of nodes, while the MPCR algorithm can consider any node in the network to be in the CT blocks, which constitute the route. Thus, the MPCR algorithm reduces the required transmitted power more than the CASNCP algorithm.

Fig. 3 depicts the required transmitted power per route by the different routing algorithms for different number of nodes at $p_{o}^{S}=0.95$ and $\eta_{o}=1.9 \mathrm{~b} / \mathrm{s} / \mathrm{Hz}$. As shown, the required transmitted power by any routing algorithm decreases with the

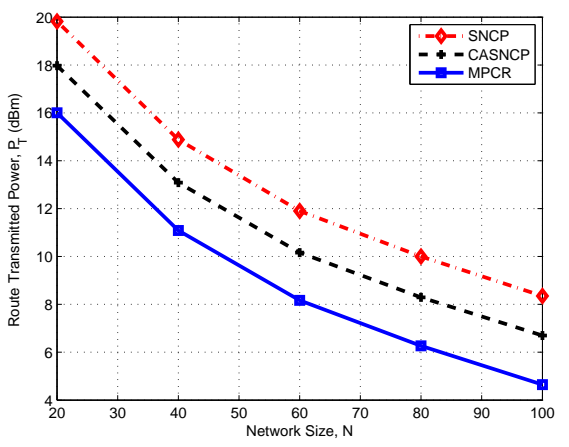

Fig. 3. Required transmitted power per route versus the number of nodes for $\eta_{o}=1.9 \mathrm{~b} / \mathrm{s} / \mathrm{Hz}$ and $\alpha=4$ in a $200 \times 200$ grid.

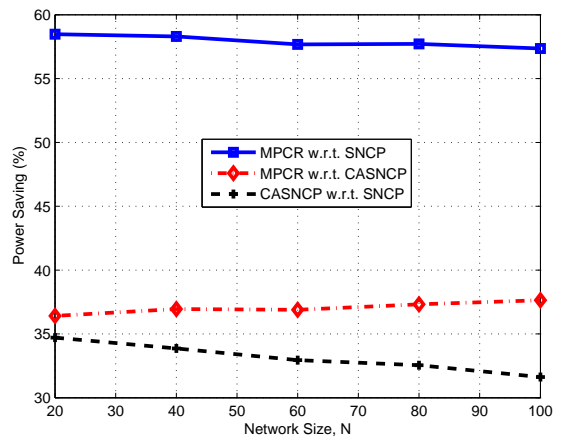

Fig. 4. Power savings per route versus the number of nodes for $\eta_{o}=1.9$ $\mathrm{b} / \mathrm{s} / \mathrm{Hz}$ and $\alpha=4$ in a $200 \times 200$ grid.

number of nodes. Intuitively, the higher the number of nodes in a fixed area, the closer the nodes to each other, the lower the required transmitted power between these nodes, which results in lower required end-to-end transmitted power.

We also calculate the power saving ratio as a measure of the improvement of the MPCR algorithm. The power saving of scheme 2 with respect to scheme 1 is defined as

$$
\text { Power Saving }=\frac{P_{T}(\text { Scheme } 1)-P_{T}(\text { Scheme } 2)}{P_{T}(\text { Scheme } 1)} \%,
$$

where $P_{T}($.$) denotes the total transmitted power for certain$ scheme. Fig. 4 depicts the power saving of the different routing algorithms with respect to each other. The shown curves are obtained through direct substitutions of the required transmitted power by each algorithm in (23). At $N=100$ nodes, $p_{o}^{S}=0.95$, and $\eta_{o}=1.9 \mathrm{~b} / \mathrm{s} / \mathrm{Hz}$, the power savings of MPCR algorithm with respect to the SNCP and CASNCP algorithms are $57.36 \%$ and $37.64 \%$, respectively. In addition, the power saving of the CASNCP algorithm with respect to the SNCP algorithm is $31.62 \%$.

Fig. 5 depicts the required transmitted power per route of the different routing algorithms with respect to the desired bandwidth efficiency for $N=20$ and $N=100$ nodes. As mentioned with respect to Fig. 2, the proposed MPCR algorithm requires the least transmitted power per route. In addition, we calculate the power saving of the MPCR algorithm as in (23). At $R_{o}=6 \mathrm{~b} / \mathrm{s} / \mathrm{Hz}$ and $N=100$ nodes, the MPCR algorithm reduces the transmitted power by $50.22 \%$ 


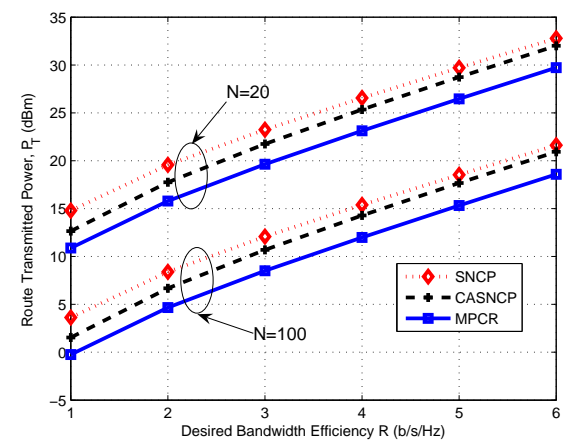

Fig. 5. Required power per route versus the desired bandwidth efficiency for $N 0=-70 \mathrm{dBm}, p_{d}^{S}=0.95 \mathrm{~b} / \mathrm{s} / \mathrm{Hz}$, and $\alpha=4$ in a $200 \times 200$ grid.

and $41.79 \%$ with respect to the SNCP and the CASNCP algorithms, respectively.

In Fig. 6, the average number of hops in each route, constructed by the different routing algorithms, is shown versus the number of nodes in the network. For the cooperative transmission mode, the average number of hops is defined as

$$
h^{C}=1 \cdot \operatorname{Pr}(\phi)+2 \cdot \overline{\operatorname{Pr}(\phi)}=2-\operatorname{Pr}(\phi),
$$

and the average number of hops for the direct transmission mode is one. As shown, the routes constructed by either the CASNCP or the MPCR algorithms consist of number of hops that is less than the routes constructed by the SNCP algorithm. Moreover, the average number of hops increases with $N$ as there are more available nodes in the network, which can be employed to reduce the transmitted power. In this section, we have illustrated using the numerical results the power savings of our proposed MPCR algorithm with respect to the SNCP and CASNCP algorithms.

\section{Conclusions}

In this paper, we have investigated the impacts of the cooperative communications on the minimum-power routing problem in wireless networks. For a given source-destination pair, the optimum route requires the minimum end-to-end transmitted power while guaranteeing certain throughput. We have proposed the MPCR algorithm, which applies the cooperative communication while constructing the route. The MPCR algorithm constructs the minimum-power route using any number of the proposed cooperation-based building blocks, which require the least possible transmitted power. For comparison issues, we have also presented the CASNCP algorithm, which is similar to most of the existing cooperative routing algorithms. The CASNCP algorithm first constructs the conventional shortest-path route then applies a cooperativecommunication protocol upon the established route. From the simulation results, the power savings of the MPCR algorithm with respect to the shortest-path and CASNCP algorithms are $57.36 \%$ and $37.64 \%$, respectively.

\section{REFERENCES}

[1] L. M. Feeney and M. Nilsson, "Investigating the energy consumption of a wireless network interface in an ad hoc networking environment," in Proc. of IEEE INFOCOM, Anchorage, AK, Apr. 2001.

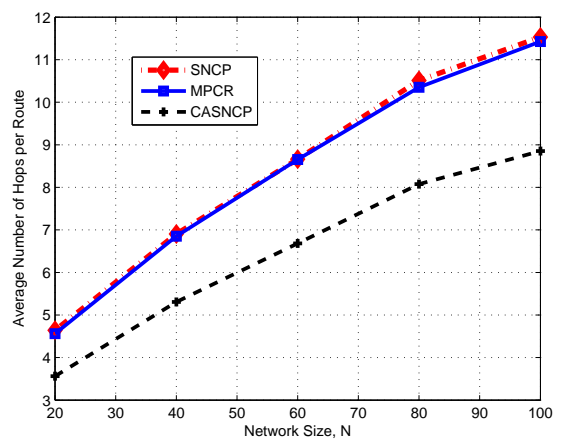

Fig. 6. Average number of hops per route versus the number of nodes for $\eta_{o}=1.9 \mathrm{~b} / \mathrm{s} / \mathrm{Hz}$ and $\alpha=4$ in a $200 \times 200$ grid .

[2] T. M. Cover and A. El Gamal, "Capacity theorems for the relay channel," IEEE Trans. Info. Theory, vol. 25, no. 5, pp. 572-584, Sept. 1979.

[3] J. N. Laneman, D. N. C. Tse, and G. W. Wornell, "Cooperative diversity in wireless networks: efficient protocols and outage behaviour," IEEE Trans. Inform. Theory, vol. 50, pp. 3062-3080, Dec. 2004.

[4] J. N. Laneman and G. W. Wornell, "Distributed space-time coded protocols for exploiting cooperative diversity in wireless networks," IEEE Trans. Inform. Theory, vol. 49, pp. 2415-2525, Oct. 2003.

[5] A. Sendonaris, E. Erkip, and B. Aazhang, "User cooperation diversityPart I: system description," IEEE Trans. Comm., vol. 51, pp. 1927-1938, Nov. 2003.

[6] A. Sendonaris, E. Erkip, and B. Aazhang, "User cooperation diversityPart II: implementation aspects and performance analysis," IEEE Trans. Comm., vol. 51, pp. 1939-1948, Nov. 2003.

[7] A. S. Ibrahim, A. K. Sadek, W. Su, and K. J. Ray Liu, "Cooperative communications with partial channel state information: when to cooperate?," in Proc. of IEEE Global Telecommunications Conference (Globecom'05), pp. 3068 - 3072, vol.5, Dallas, TX, 28 Nov.-2 Dec. 2005.

[8] A. S. Ibrahim, A. K. Sadek, W. Su, and K. J. Ray Liu, "Relay Selection in multi-node cooperative communications: When to cooperate and whom to cooperate with?," in Proc. of IEEE Global Telecommunications Conference (Globecom'06), San Francisco, CA, Nov. 2006.

[9] A. K. Sadek, W. Su, and K. J. Ray Liu, "A class of cooperative communication protocols for multi-node wireless networks", in Proc. of IEEE International Workshop on Signal Processing Advances in Wireless Communications (SPAWC'05), pp. 560 - 564, New York, NJ, June 2005.

[10] A. K. Sadek, Z. Han, and K. J. Ray Liu, "A distributed relay-assignment algorithm for cooperative communications in wireless networks", in Proc. of IEEE International Conference on Communications, Istanbul, Turkey, June 2006.

[11] A. E. Khandani, E. Modiano, L. Zheng, and J. Abounadi, "Cooperative routing in wireless networks," Chapter in Advances in Pervasive Computing and Networking, Kluwer Academic Publishers, Eds. B. K. Szymanski and B. Yener, 2004.

[12] Z. Yang, J. Liu, and A. Host-Madsen, "Cooperative routing and power allocation in ad-hoc networks," in Proc. of IEEE Global Telecommunication Conference, Globecom, Dallas, TX, Nov. 2005.

[13] F. Li, K. Wu, and A. Lippman, "Energy-efficient cooperative routing in multi-hop wireless ad hoc networks," in Proc. of IEEE International Performance, Computing, and Communications Conference, pp. 215 222, Phoenix, AZ, Apr. 2006.

[14] M. Sikora, J. N. Laneman, M. Haenggi, D. J. Costello, and T. E. Fuja, "Bandwidth- and power-efficient routing in linear wireless networks," IEEE Trans. Inform. Theory, vol. 52, pp.2624-2633, Jun. 2006.

[15] J. Luo, R. S. Blum, L. J. Greenstein, L. J. Cimini, and A. M. Haimovich, "New approaches for cooperative use of multiple antennas in ad hoc wireless networks," in Proc. of IEEE 60th Vehicular Technology Conference, vol. 4, pp. 2769 - 2773, Los Angels, CA, Sept. 2004.

[16] D. Bertsekas and R. Gallager, Data networks, 2nd ed., Prentice Hall, 1991.

[17] L. Zheng and D. N. C. Tse, "Diversity and multiplexing: a fundamental tradeoff in multiple-antenna channels," IEEE Trans. on Info. Theory, vol. 49, pp. 1073 - 1096, May 2003.

[18] J. G. Proakis, Digital communications, 4th ed., McGraw-Hill, 2000.

[19] D. G. Brennan, "Linear diversity combining techniques," Proceedings of the IEEE, vol. 91, no. 2, pp. 331-356, Feb. 2003. 